Check for updates

Cite this: Mater. Adv., 2021, 2, 6928

Received 17th June 2021 Accepted 21st September 2021

DOI: 10.1039/d1ma00525a

rsc.li/materials-advances

\title{
Synthetic polymer-derived single-network inks/bioinks for extrusion-based 3D printing towards bioapplications
}

\begin{abstract}
Sonu Kumar (iD)
Three-dimensional (3D) printing, also known as the additive manufacturing technique has revolutionized the field of manufacturing with great impact as compared to the other traditional methods. This technique has shown a steep increase in popularity over the past decade due to its benchmark capabilities of fabricating new and complex 3D constructs, especially towards tissue engineering and regenerative medicine. Among the currently applied 3D printing techniques, extrusion-based 3D printing has garnered particular attention for the employment of ink/bioink materials to enable on-demand personalized fabrication due to its low cost, broad utility for various materials, and ease of controlled printability. However, there is still a lack of diversity in the ink materials with their optimized degradation rate, rheology, and bioactivity for precisely fabricating complex and self-supported cell-laden 3D printed constructs. Therefore, the development of an array of such new materials is a major challenge for synthetic polymer chemists, material scientists and biomedical researchers for widening the future applicability of 3D (bio)printing. This review aims to summarize the recent advances in the rational design and development of ink/bioink materials based on synthetic polymers as single network precursors due to their great opportunity to tune their physicochemical and mechanical properties in order to design and mimic in-human 3D tissue scaffolds with shape retention for both hard and soft tissues.
\end{abstract}

\section{Introduction}

The 3D printing-based computer-aided additive manufacturing technique has emerged as a revolutionary manufacturing method and is currently being considered at the forefront of research in material sciences and biomedical engineering. ${ }^{1,2}$ $3 \mathrm{D}$ printing is a promising method for the future era of personalized medicine via the layer-by-layer fabrication of patient-specific, customizable medical devices, organs, tissues, and other bio-systems, mimicking their native counterparts with complex and heterogeneous structures. ${ }^{3,4}$ In the endeavours for such biofabrication, the selection of printing technology, design and materials are critically important for maintaining the structureproperty-processing relationships in the $3 \mathrm{D}$ printed constructs. ${ }^{5,6}$ To date, there has been considerable development in the engineering of different types of 3D printing devices ${ }^{7-11}$ and increasing their printing affordability and reliability such as having tight control over the printing speed, higher resolutions, and multicomponent fabrications. ${ }^{12,13}$ The development and

Department of Applied Sciences (Chemistry), Punjab Engineering College (Deemed to be University), Sector 12, Chandigarh, 160012, India.

E-mail:sonu.kumar@pec.edu.in optimization of the printable ink materials are required to achieve the desired properties including physico-mechanical properties, rheological properties and biofunctionality to obtain self-supporting functional and high-strength products, depending on the applications. ${ }^{14,15}$ Although there exist different ink materials ranging from polymers to composites and ceramics, ${ }^{16}$ there is still the need for diversity in 3D printable ink materials. On the other hand, the preparation of 3D printable cell-laden ink materials (i.e. bioinks) has attracted a great deal of interest from researchers in recent years, ${ }^{17}$ and has been considered as one of the most advanced tools for finding new avenues in tissue engineering, ${ }^{18}$ regenerative medicine, ${ }^{19}$ drug delivery, ${ }^{20}$ cell therapy, ${ }^{21}$ etc. To mimic the biocomplexity and heterogeneity of natural tissues in the 3D printed constructs on various scales, the development of cellular embedded ink materials with "printability" (e.g., with optimized printing process, speed, and resolution are critically important $)^{22,23}$ is challenging due to the demand for various features depending on the application envisioned as discussed here. Cells are encapsulated within such ink materials, and therefore all the components of the inks such as the functionalized polymer-based main ingredient along with other applied precursors such as catalysts, cross-linker, drugs, bioactive 
molecules (e.g. peptide sequences, ${ }^{24}$ growth and differentiation factors, etc. ${ }^{25,26}$ ) should be biocompatible in order to maintain the cell survivability throughout the printing process, as well as in the final printed article. The ink materials should protect the cells and maintain their sensitivity to enable the survival of cells during the printing process for the bio-fabrication, and the desired cellular functions (e.g., cell growth and proliferation, adhesion, and differentiation) should be preserved in the fabricated structures. ${ }^{27}$ Further, the post-extrusion structural and functional integrity in the product should be maintained by standardizing different parameters such as pressure, temperature, $\mathrm{pH}$, or light to avoid cell resistance. ${ }^{28,29}$ Finally, the materials should be biodegradable with controlled degradation kinetics and their waste compounds and intermediates should not have any toxic effects.

In the effort to develop ink materials for 3D printing with desired mechanical and biological properties, two major categories of material precursors have been adopted: (i) natural polymers (biomaterials), and (ii) synthetic polymers. Natural polymers, such as polysaccharides and proteins, as typically extracted from plants, animals, bacteria, cells, etc., are currently widely applied materials as network precursors in bioink formation due to their greater biocompatible nature and higher cellular proliferation rates in comparison to synthetic polymers. ${ }^{30}$ Note that in many reports of bioink formulation natural polymers were artificially modified with functional groups, for instance, to enable network crosslinking, however, such artificial biomaterials have been considered in this review in the category of natural polymers only. ${ }^{31,32}$ Naturally derived polymers are often associated with some disadvantages that can lead to challenges in their utility for biofabrication as follows: (1) batch-to-batch variations, which can lead to complications due to their variable printabilty and the issue of the reproducibility of constructs, and the cellular sensitivity to such variations; (2) tuning the structures, solubility, viscosity and other properties of biopolymers remain challenging since their functionalization is often more difficult; (3) fast biodegradability rate, which is often not suitable. On the other hand, synthetic polymers can overcome these limitations of natural polymers along with the opportunity to be tailored with specific physical, chemical, and biological properties and can further lead to the synthesis of a broad library of new materials for (bio)inks. ${ }^{33}$ Synthetic polymers also further offer the ability to tune functional properties and rheological behaviours, like the printability and mechanical integrity of the inks, through monomer selection, architecture control, and post-polymerization functionalization opportunities. ${ }^{34,35}$ Therefore, synthetic polymers have great potential to be exploited as future ink materials of 3D printing for shaping into advanced and highly customisable architectures suitable for medical devices and tissue scaffolds, including both hard and soft tissues. ${ }^{36,37}$ However, a careful selection of synthetic polymer has to be made in order to maintain biocompatibility and biodegradation issues. For instance, poly(ethylene glycol) (PEG) corresponds is a synthetic polymer that has been approved by the Food and Drug Administration (FDA) and shows good water solubility as well as biocompatibility. ${ }^{38,39}$ Poly(lactic-co-glycolic) acid (PLGA), polylactic acid (PLA), and polycaprolactone (PCL) are also FDA-approved synthetic polymers for their application in medical devices and are biocompatible and biodegradable in nature. ${ }^{40,41}$ Therefore, the utilization of synthetic polymers as ink materials can provide advancement in the 3D printing technology with obtaining the highly defined (bio)fabricated constructs with better control size, resolutions, integrity and features as compared to the traditional manufacturing techniques. Gupta et al. exemplified the combination of 3D printing technique with functional plasmonic nanomaterials for exhibiting programmable release from the printed multiplexed array of stimuli-responsive capsules having shells comprised of PLGA polymer within hydrogel matrices. ${ }^{42}$ In 2019, Camacho and Busari et al. investigated RGDS(biotin)PCL and RGES(azide)-PCL-based peptide-polymer conjugates for the controlled fabrication of spatially functionalized $3 \mathrm{D}$ printed biodegradable scaffolds by using multiple printer heads, which displayed a significant enhancement in NIH 3T3 fibroblast adhesion on the 3D-printed fiber surface of (RGDS(biotin))-PCL. ${ }^{43}$ Another example of a PCL-based 3D printed scaffold was documented by Rashad et al., where it was subsequently coated with cellulose nanofibrils that significantly improved the hydrophilicity and protein absorption of the PCL scaffold surface, and further enhanced the cellular response (attachment, proliferation, and osteogenic differentiation) of mesenchymal stem cells (hBMSCs) in vitro for the potential application of such 3D printed PCL scaffolds towards bone tissue regeneration in vivo. ${ }^{44}$ In contrast to the recent burgeoning advancements in the $3 \mathrm{D}$ (bio)printing technique, the focus of this review is to highlight the examples and development of the (bio)ink materials based on synthetic polymers as a singlenetwork precursor (or major component) employed for the extrusion-based 3D printing technique towards bioapplications since, to the best of our knowledge, this area of interest has not been previously reviewed. Note, that the formulation of (bio)ink materials based on only natural polymers or combined natural and synthetic polymers is out of the scope of this review article. The existing challenges associated with 3D biofabrication have been also discussed in this article to motivate synthetic polymer chemists to address the current demand for new and advanced (bio)ink materials, which would further help to widen the scope of this unique and advanced technique of extrusion-based 3D printing.

\section{The extrusion-based 3D printing technique}

Extrusion-based 3D printing is the commercially available and most common rapid prototyping technique due to its affordability, versatility, and compatibility with a wide selection of ink materials usable for biofabrication and tissue regeneration ranging from small vessels to large constructs/organs. ${ }^{45,46}$ Here, the inks are extruded through a micronozzle of extrusion head and controlled by either an endless screw, pneumatic 


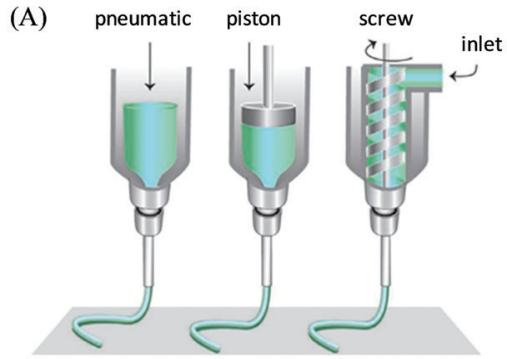

(B)

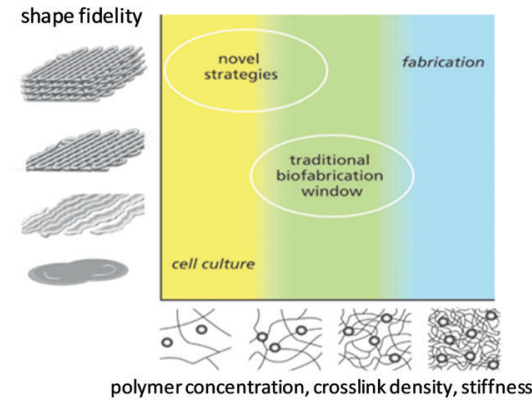

$\begin{array}{llll}\text { (C) Pre-crosslink } & \text { (2) Post-crosslink } & \text { (3)-situ-crosslink }\end{array}$

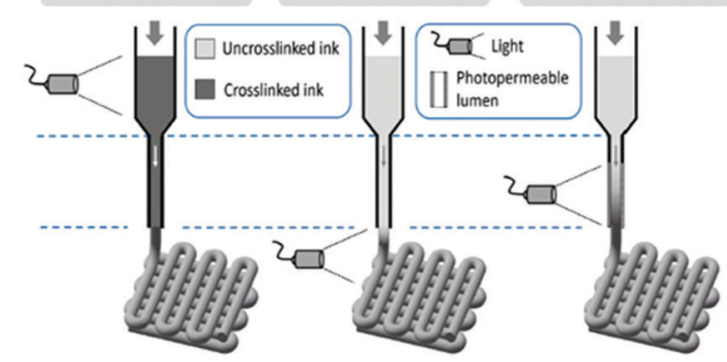

(D)

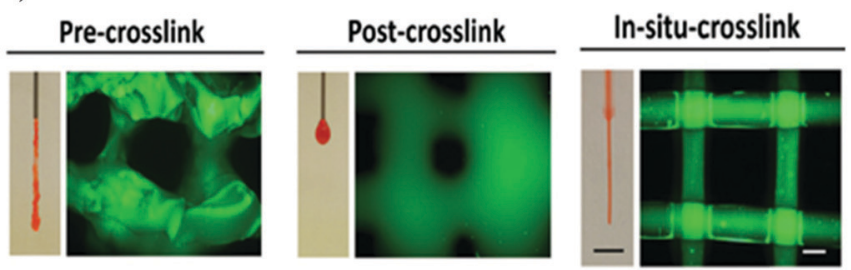

Fig. 1 (A) Schematic illustration of the working principle of extrusion-based 3D printing techniques, and (B) the concept of the biofabrication window to improve the printability of polymeric hydrogel bioinks. Adapted with permission from ref. 47. Copyright 2013 John Wiley and Sons. (C) Schematic illustration of different bioprinting approaches for photo-crosslinkable inks via crosslinking before (pre-crosslinking), after (post-crosslinking), or during (in situ crosslinking) extrusion. (D) Representative images of the extruded material from nozzles and the lattice structure of corresponding printed constructs. Reproduced with permission from ref. 52. Copyright 2017 John Wiley and Sons.

pressure, or a mechanical piston (Fig. 1A). ${ }^{47,48}$ The 3D objects are constructed via the blueprints from a computer-aided design (CAD) file by direct-ink-writing in a layer-by-layer fashion, and controlling the $x y z$ stage of the deposition path of the print-heads. ${ }^{49}$ Extrusionbased 3D printers can be successfully engineered under sterile conditions with nozzle diameters and multiple extrusion heads providing the unique advantage of depositing different types of layers in the final printed article via delivering multiple (bio)ink materials with varying components, cells, and cellular densities (Fig. 1B). ${ }^{8,50}$ To successfully enable the "printability" of an ink, a suitable viscosity should be achieved, typically by inducing either an external shear force (i.e. shear-thinning property) or precrosslinking to enable its extrusion from the nozzle; however as the external mechanical stress is removed post-extrusion, a rapid filament shape retention with efficient recovery of mechanical properties (i.e. self-healing properties) is desired to keep entire and self-supported 3D printed structures with no structural collapse. ${ }^{51}$ For instance, UV irradiation (or heating) can be applied for such shape retention to the tip of the printing nozzle or directly over the printed structure on the deposition plate. Burdick and coworkers developed a general extrusion-based bioprinting method of "in situ crosslinking" for photo-crosslinkable non-viscous hydrogel inks (such as from $5 \mathrm{wt} \%$ methacrylated hyaluronic acid (MeHA)) via introducing light through a photopermeable capillary, which enables their simultaneous extrusion and crosslinking, prior to deposition (Fig. 1C and D).$^{52}$ The printed constructs depicted the uniform filament formation along with the high viability of the encapsulated cells. Another fascinating approach that has gained significant interest recently is extrusion-based 3D bioprinting using suspension baths, illustrating their ability to suspend and completely encapsulate the extruded filament materials. ${ }^{53}$ Such secondary support provides the flow restriction of the bioink and triggers the cross-linking (physically or chemically) immediately after the deposition, and enables the printed construct with improved resolution and controlled heterogeneity ${ }^{54}$ Feinberg and co-workers presented such an approach as Freeform Reversible Embedding of Suspended Hydrogels (FRESH) printing, where a thermoreversible support bath $\left(\mathrm{CaCl}_{2}\right)$ was utilized to embed a soft hydrogel (based on alginate) to exemplify the construction of a bioprinted full-sized human heart model. ${ }^{55}$ Recently, a co-axial extrusion-based 3D bioprinting approach has also been introduced to enable the simultaneous printing of bioink along with crosslinker solutions in a coaxial system to promote gelation during extrusion. ${ }^{56}$ Khademhosseini, Dentini, and co-workers demonstrated a coaxial needle extrusion system where the internal needle carried the flow of cell-laden bioink materials (based on alginate and gelatin methacroyl) and the external needle was designed for the simultaneous flow of ionic crosslinking solution $\left(\mathrm{CaCl}_{2}\right)$ to generate the ionically crosslinked hydrogel microfibers at the tip of the dispensing system, which was further secondarily crosslinked covalently via exposure to UV light. ${ }^{57}$

\section{Hydrogel inks/bioinks based on synthetic polymers as single-network precursors}

Hydrogels are highly hydrated three-dimensional polymeric networks and have been exploited for various biomedical applications, such as scaffolds for tissue engineering, and drug 
delivery. ${ }^{58,59}$ Hydrogel-based ink materials can provide a perfect soft material for soft tissue engineering since creating a soft tissue is more challenging due to the demand for features like high elasticity, flexibility, viscosity, and inter-layer adhesion. ${ }^{60,61}$ From a biological point of view, hydrogels having a high water content can be considered as an ideal candidate for cell-laden bioink materials for extrusion-based 3D printing because they can closely simulate the native extracellular matrix (ECM) microenvironments having an aqueous 3D environment, and provide cell survivability with retained rounded morphology and homogeneous encapsulation during the extrusion process, and thereafter promote new tissue formation and functioning in 3D space. $^{18,62}$ However, the fabrication of hydrogel-based scaffolds or cell-laden constructs with shape fidelity remains challenging, and there is a current demand for optimization of the mechanical properties of the hydrogel materials within a biofabrication window (Fig. 1B). ${ }^{47}$ On the one hand, the soft hydrogels, which are well suited for cells, are disadvantageous for fabrication processes to achieve the constructs with maintained predesigned structural fidelity. On the other hand, the stiff hydrogels, which can typically provide shape fidelity, can affect the cellular functioning by limiting the migration and proliferation of the cells within the dense polymeric network.

In this context, the utilization of synthetic polymers as hydrogel ink materials can tailor the mechanics by maintaining adequate viscoelastic characteristics and integrity, ${ }^{63,64}$ including self-healing and shear-thinning properties, and also provide opportunities for optimization in its functionality, and degradation kinetics of the printed products. ${ }^{65,66}$ In general, hydrogel ink formulation requires a specific viscous polymeric solution that can immediately form high networking post-printing either by the physical or chemical cross-linking of polymers. ${ }^{67}$ Prestwich and coworkers described the formation of extrudable hydrogels with suitable rheological properties for the bioprinting of vessel-like constructs derived from tetrahedral polyethylene glycol tetracrylates via co-crosslinking thiolated hyaluronic acid and gelatin derivatives. $^{68}$ Joas et al. investigated the hydrogel formulation for extrusion-based 3D printing comprised of the diacrylate of PEG, the anionic and cationic monomers 3-sulfopropyl acrylate and [2-(acryloyloxy)ethyl]trimethylammonium chloride, respectively, via UV irradiation-based photo-polymerization. ${ }^{69}$ The biodegradable cross-linked hydrogel network (using visible light stimulus) was further illustrated by $\mathrm{Xu}$ et al. based on a single-component

(A)
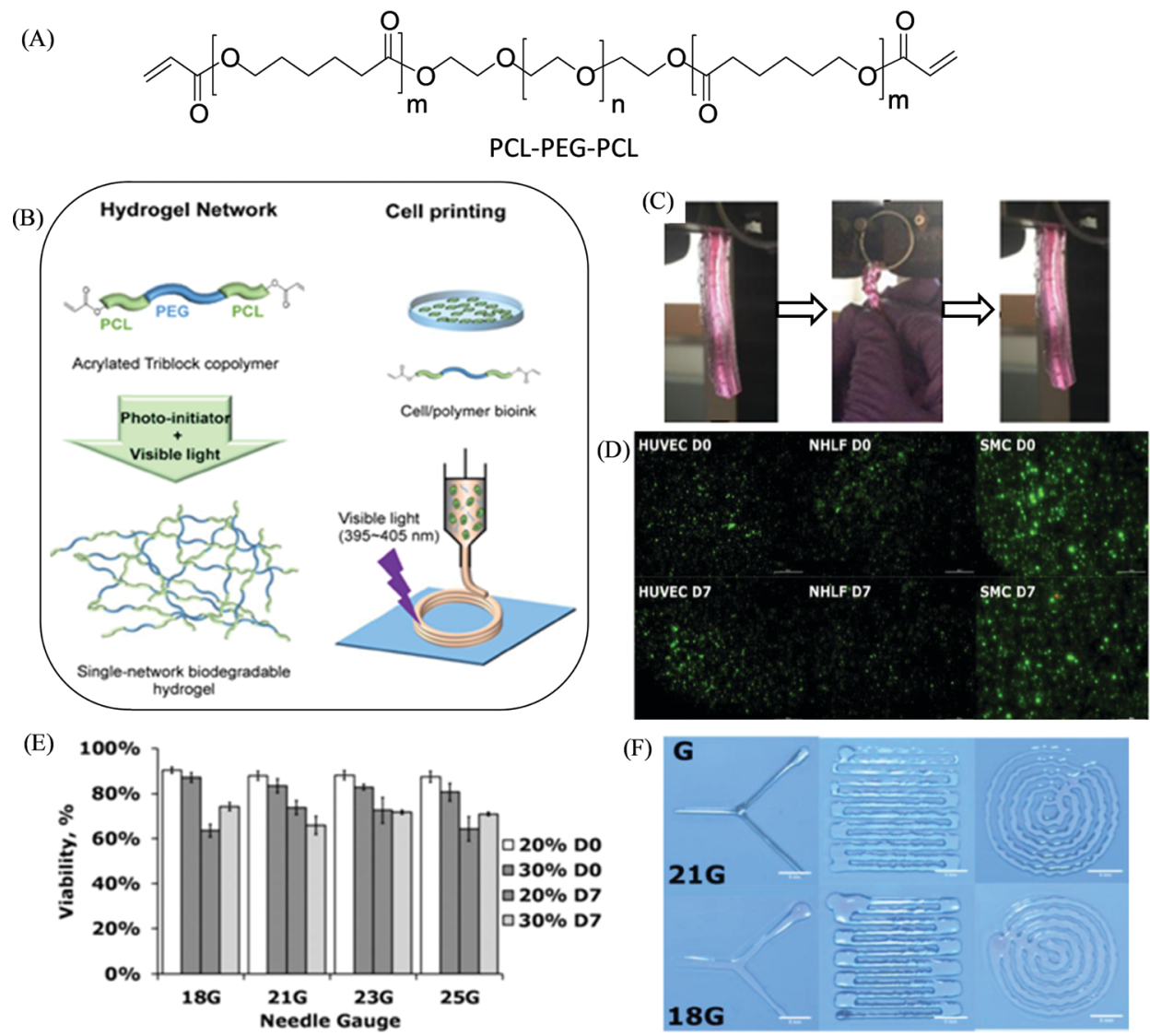

Fig. 2 (A) Chemical structure of the acrylated PCL-PEG-PCL triblock copolymer. (B) The preparation of a visible-light-induced cross-linked singlenetwork biodegradable hydrogel having high elasticity for bioprinting with various human cells. (C) Photographs illustrating the mechanical properties of the PCL-PEG-PCL hydrogel upon twisting for four cycles and recovering after release. ( $D$ and E) Live/dead cell assay and cellular compatibility, respectively, of different cell types in the printed 10\% PCL-PEG-PCL hydrogel carried out immediately after gelation (D0) and after 7 days in culture (D7) (scale bar: $500 \mu \mathrm{m}$ ). (F) Printed sample shapes by using different needle sizes (scale bar: $5 \mathrm{~mm}$ ). Adapted with permission from ref. 70. Copyright 2018 American Chemical Society (ACS). https://pubs.acs.org/doi/10.1021/acsami.8b01294 (for further permissions related to the material excerpted, readers are directed to the ACS). 
precursor of diacrylated polycaprolactone-poly(ethylene glycol)polycaprolactone (PCL-PEG-PCL) triblock copolymer, which was synthesized (theoretical block length $2 \mathrm{k}-20 \mathrm{k}-2 \mathrm{k}$ ) via ringopening polymerization followed by acryloyl group insertion (Fig. 2).$^{70}$ The hydrogels were composed of PEG and PCL-based well-known biocompatible synthetic materials and therefore, the hydrogels exhibited support for cellular growth with good survival rates (in vitro) of the encapsulated 3T3 fibroblasts of over $90 \%$ from day 1 and day 3 of culture. The hydrogels possessed good mechanical properties with high flexibility and elasticity upon stretching, compressing, and twisting. The viscosities of the obtained hydrogel precursors were optimized to prepare cell/polymer bioink (with PCL-PEG-PCL at $10 \%$ of polymer concentration) based on different types of human cells such as human umbilical vein endothelial cells (HUVEC), human aortic smooth muscle cells (SMC), and neonatal human lung fibroblasts (NHLF). 3D bioprinting was performed to create cell-gel constructs upon visible-light exposure, exhibiting their high cellular viability over $83 \%$ across all three cell types, within the constructs after 7 days in culture printing. Basic shapes and complex patterns were printed using $20 \%(\mathrm{w} / \mathrm{v})$ PCL-PEG-PCL solution, and their resolutions were maintained by changing the nozzle diameter (with 18G and 21G needle), indicating the printability of the hydrogels.

\subsection{Supramolecular polymers}

Supramolecular polymers are derived from a unique combination of supramolecular chemistry and polymer science, ${ }^{71,72}$ and have appeared as advanced smart materials for diverse applications towards energy harvesting, drug delivery, electronic devices, template synthesis, etc. ${ }^{73,74}$ Such polymeric chains involve various types of supramolecular interactions based on hydrogen bonding, $\pi-\pi$ interactions, metal-ligand coordination, interactions between ions, and host-guest interactions. ${ }^{75,76}$ Those supramolecular noncovalent interactions offer a dynamic and reversibile nature, providing an opportunity to tune the mechanical and viscoelastic behavior with improved rheological performance of supramolecular polymers, ${ }^{77}$ which makes them potential candidates for $3 \mathrm{D}$ printing-based additive manufacturing to create customizable and dynamic constructs for tissue engineering scaffolds and other biomedical applications. ${ }^{19,78,79}$ Echalier et al. documented a 3D printable bioink derived from supramolecular peptide-functionalized synthetic polymer-based hydrogels by using soft sol-gel polymerization without using photoactivation or additional organic reagents (Fig. 3). ${ }^{80}$ Hydrolysis, followed by condensation of the silylated precursors of hybrid bifunctional PEG polymer and integrin ligand (GRGDSP peptide), occurred during the extrusion-based 3D printing process (at room temperature and in physiological buffer with $\mathrm{pH}$ 7.2) resulting in a chemically cross-linked network through siloxane ( $\mathrm{Si}-\mathrm{O}-\mathrm{Si})$ bonds. The obtained hybrid PEG-peptide-based 3D scaffolds were then seeded with a mesenchymal stem cell (mMSC) culture, which showed excellent cell viability after 4 days of proliferation live/dead assay on the scaffolds, indicating the compatibility of the hybrid PEG-peptide scaffolds for cell culture.

In another example, Lorson et al. documented thermogelling supramolecular materials as a bioink for 3D bioprinting based on a series of synthetic biocompatible amphiphilic block copolymers comprised of a poly(2-methyl-2-oxazoline) (PMeOx)based hydrophilic block and poly(2-n-propyl-2-oxazine) (PnPrOzi)-based thermoresponsive segment, yielding PnPrO$\mathrm{zi}_{n}-b$-PMeOx ${ }_{n}$ (Fig. 4). ${ }^{81}$ At concentrations of $20 \mathrm{wt} \%$ and above,

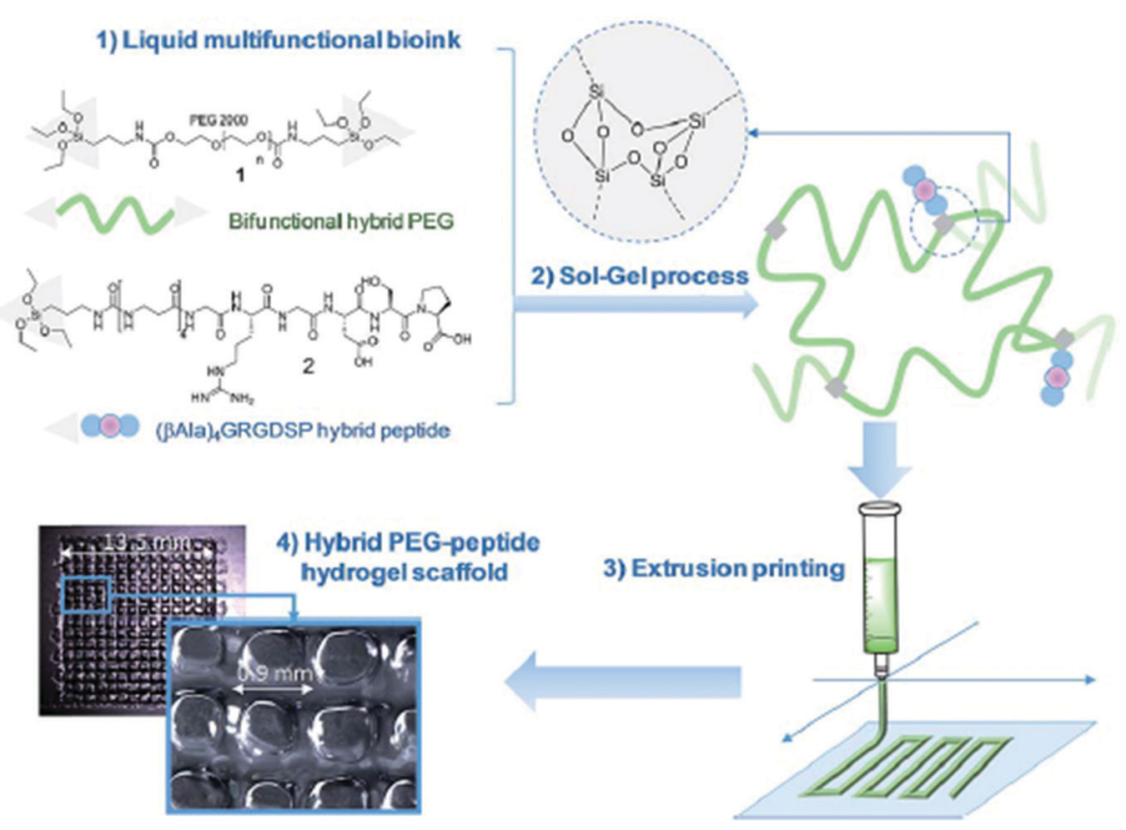

Fig. 3 Principle of sol-gel extrusion printing of PEG-peptide-based hybrid hydrogels. Hybrid silylated PEG polymers and hybrid GRGDSP peptides were dissolved in buffer to provide a multicomponent bioink that undergoes condensation during 3D printing to produce hydrogel scaffolds. Reproduced from ref. 80 with permission from The Royal Society of Chemistry. 


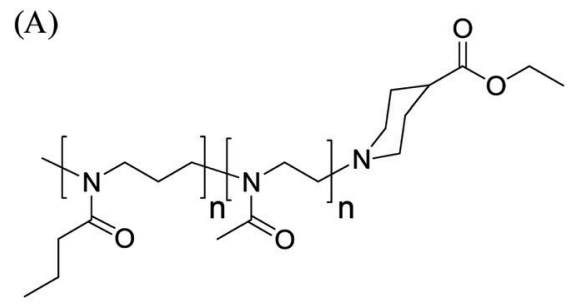

(B)

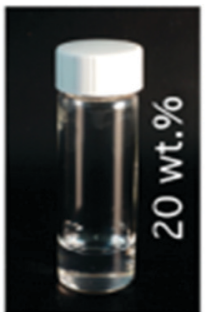

$5{ }^{\circ} \mathrm{C}$

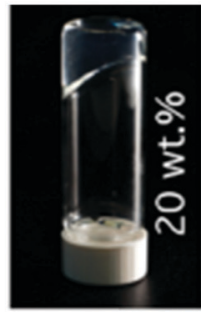

$30{ }^{\circ} \mathrm{C}$

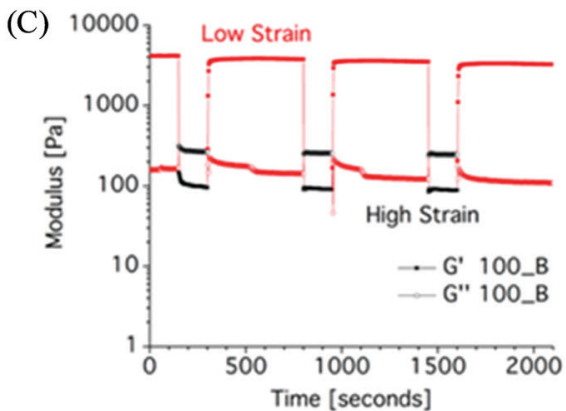

(D)

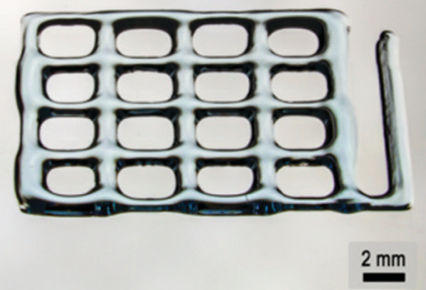

(F)
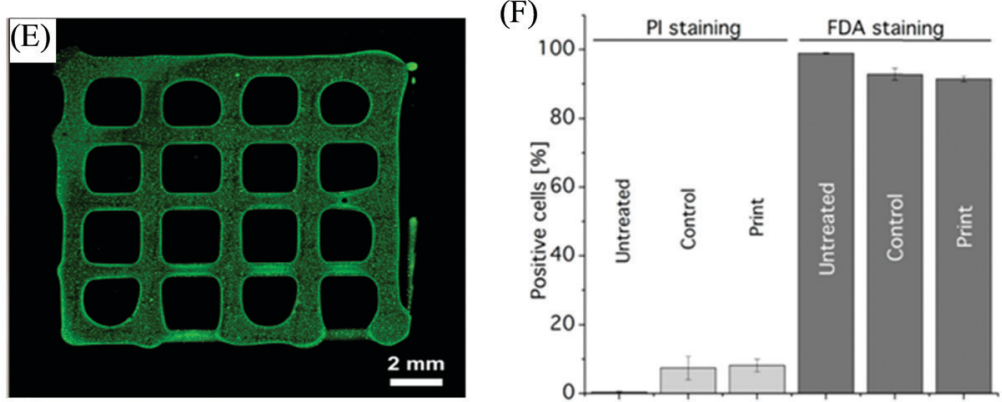

Fig. 4 (A) The chemical structure of the investigated diblock copolymer materials PnPrOzi $n-b-\mathrm{PMeOx}_{n}$. (B) Photographs of 20 wt\% concentration of PnPrOzi $1_{100}-b-\mathrm{PMeOx}_{100}\left(100 \_\mathrm{B}\right)$ at different temperatures. (C) Shear recovery at $37^{\circ} \mathrm{C}$ and $10 \mathrm{rad} \mathrm{s}^{-1}$ of (20 wt\%) $100 \_\mathrm{B}$ (low strain: 0,5\%, high strain: 150\%). (D) Light microscopy image of hydrogel 3D printed constructs, and (E) cell-loaded printed constructs. (F) FACS analysis results to illustrate the effect of the printing process on the viability of NIH $3 \mathrm{~T} 3$ fibroblast cells stained with propidium iodide (PI) and fluorescein diacetate (FDA) ("untreated" corresponds to the cells in the medium, "control" refers to the cells redispersed in the bioink but not printed, and "print" refers to the viability of cells after $24 \mathrm{~h}$ of printing). Adapted with permission from ref. 81. Copyright 2017 American Chemical Society.

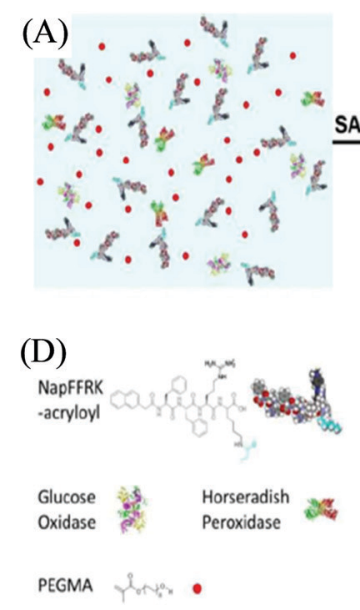

(B)

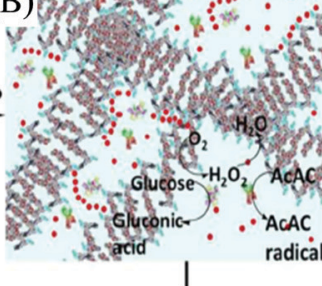

(C)

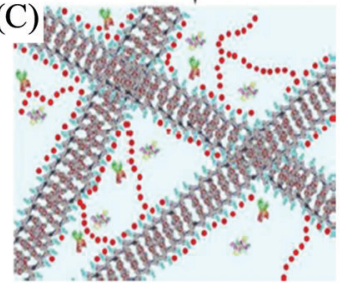

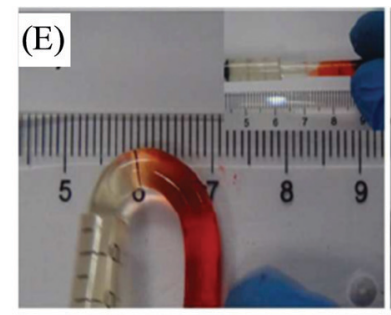
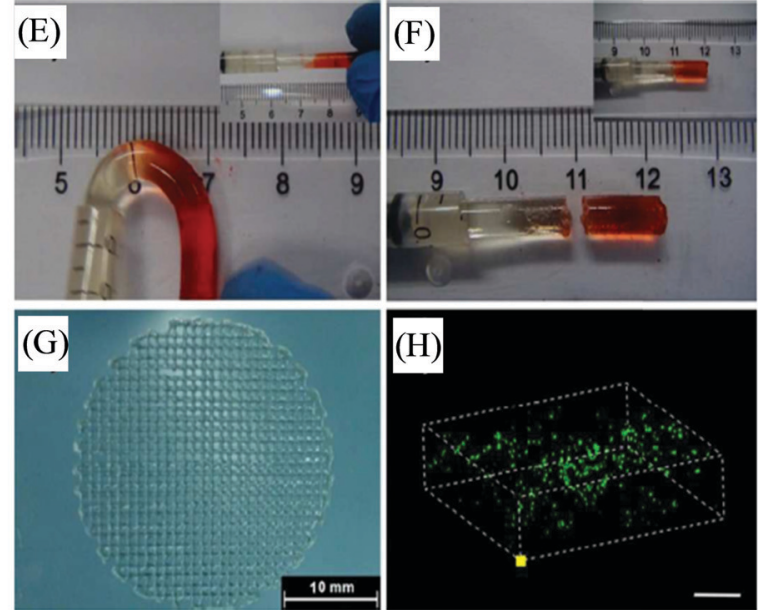

Fig. 5 (A) Schematic representation of the pre-gel solution. (B) Dual-enzyme-triggered self-assembly (SA) and simultaneous enzyme-initiated polymer reaction (EIP). (C) The hybrid hydrogel formation with the incorporated dual enzyme. (D) Schematic illustration of the peptidic hydrogelator, enzymes, and PEGMA (ACAC and glucose are omitted). (E) The self-healing of the hybrid hydrogel at the joint by placing two parts together at $3600 \mathrm{~s}$. (F) The joint cannot withstand bending by placing them together at $6000 \mathrm{~s}$. (G) 3D printed constructs. (H) A 3D stack of printed cells in the hybrid hydrogel materials stained with FDA and PI (scale bar represents $300 \mu \mathrm{m}$ ). Reproduced from ref. 83 with permission from The Royal Society of Chemistry. 


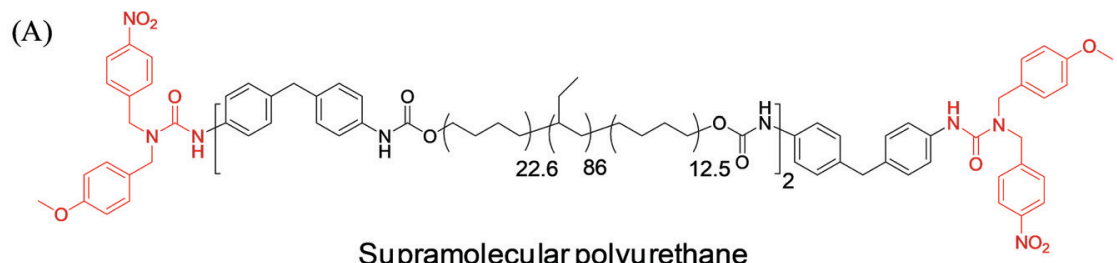

Supramolecular polyurethane

(B)

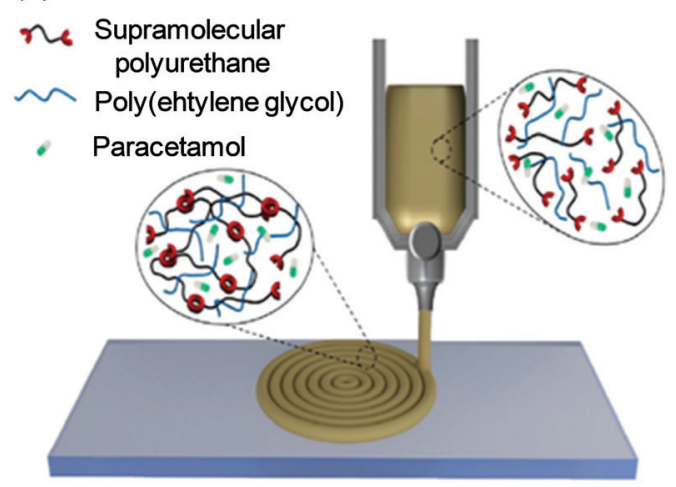

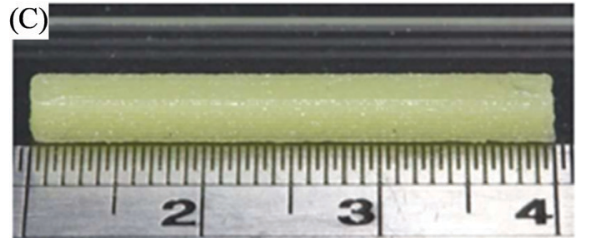

(D)

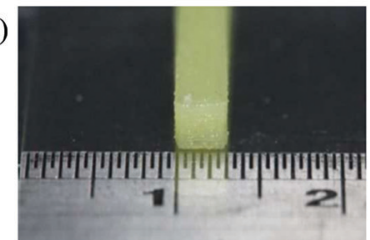

Fig. 6 (A) Chemical structure of the dynamic self-assembling supramolecular polyurethane (SPU) materials. (B) Schematic illustration of the SPU-PEGparacetamol formulation-based 3D printing of the prototype drug-release implant. (C and D) Side-on and end-on view photographs of the 3D printed bar construct from such formulation. Reproduced from ref. 86 with permission from The Royal Society of Chemistry.

the copolymers depicted thermogelation between room temperature and $37{ }^{\circ} \mathrm{C}$, and the formed hydrogels exhibited high mechanical strength with G' values more than $1 \mathrm{kPa}$, and pronounced isothermal shear thinning behavior with a rapid and complete shear recovery upon the removal of stress. Such rheological properties of the copolymer materials along with
(A)

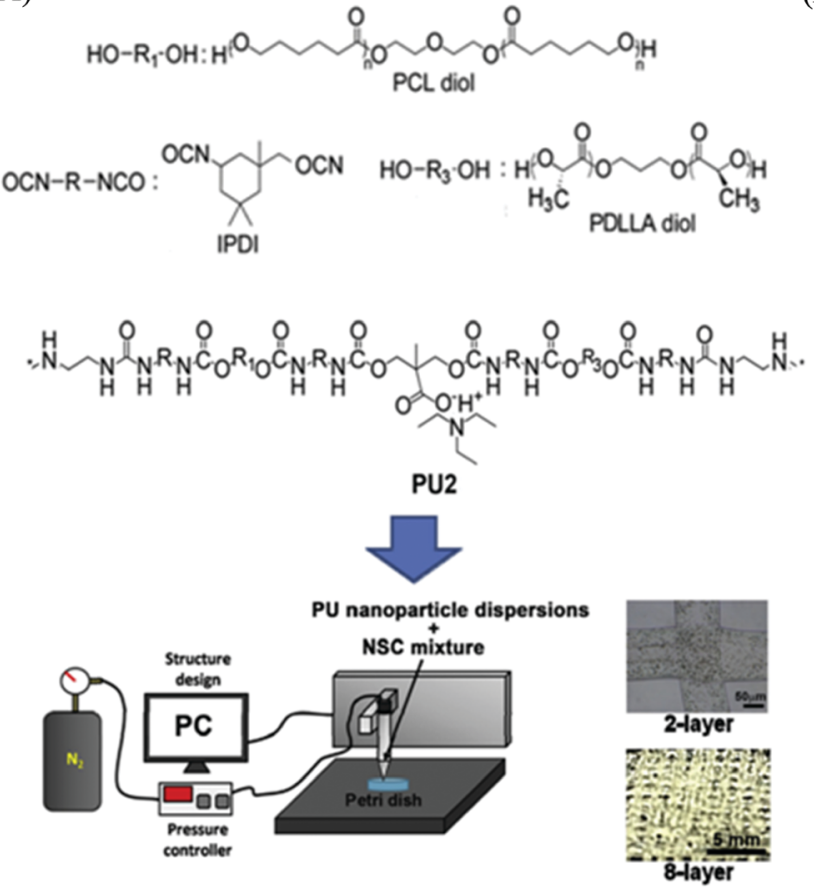

(B)
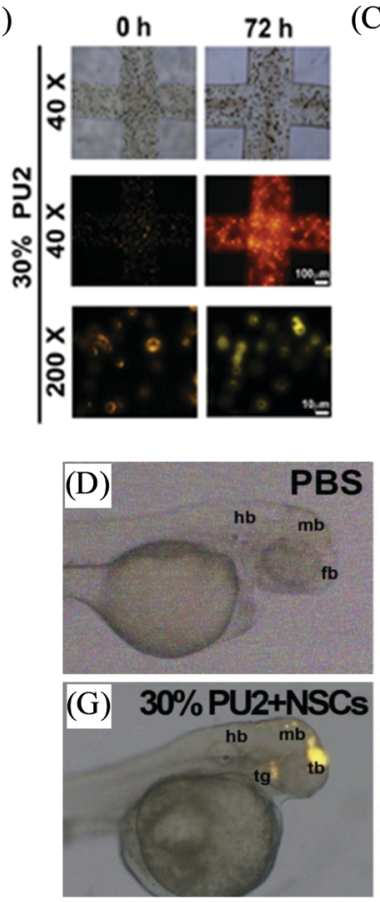

(C)
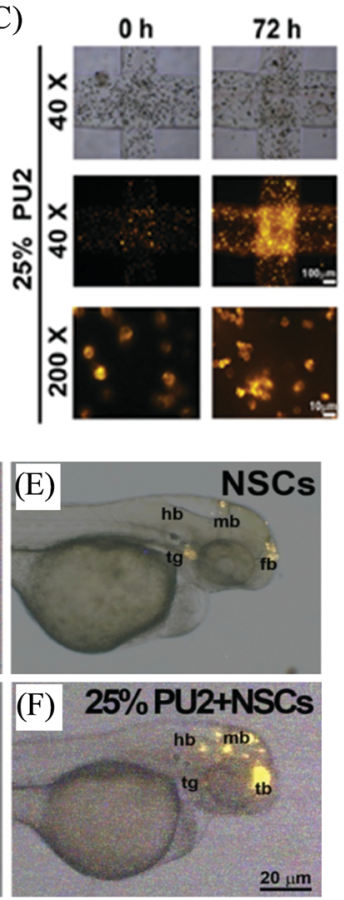

Fig. 7 (A) Schematic representation depicting the chemical structure of polyurethane (PU), and the 3D bioprinting process for creating constructs based on the neural stem cell (NSC)-laden PU hydrogel. (B and C) Photographs of NSCs (labeled with PKH26) in the 3D printed stacking fibers at $0 \mathrm{~h}$ and $72 \mathrm{~h}$ corresponding to 25 and 30\% PU2 hydrogels bionks, respectively. (D-G) The distribution of NSCs (labeled with PKH26) in the zebrafish embryos at $48 \mathrm{~h}$ post-fertilization, injected with PBS, NSCs, and NSC-laden PU2 hydrogels of 25 and 30\%, respectively. Adapted with permission from ref. 95. Copyright 2015 Elsevier. 
their obtained cytocompatibility, enabled their suitability for extrusion-based 3D bioprinting to afford cell-loaded constructs. ${ }^{82}$ The homogeneous cell distribution was observed within the entire printed constructs generated from NIH 3T3 fibroblasts encapsulated PnPrOzi- $b$-PMeOx-based bioinks and no negative effects on the cell viability were observed by the dispensing process, with cytocompatiblity (91.5 $\pm 0.8 \%$ ) measured $24 \mathrm{~h}$ post-printing, which was comparable to the viability of the control cells incorporated in the material but not printed $(92.8 \pm 1.7 \%)$, and untreated cells in the medium $(98.9 \pm 0.18 \%)$.

The one-pot fabrication of printable supramolecular polymer-based self-healing hydrogels was demonstrated by Wei et al., derived from guanidinium-based oligopeptide via the dual-enzyme-triggered self-assembly and simultaneous enzyme-initiated polymerization approach (Fig. 5). ${ }^{83}$ The ink material for such a system was comprised of the mixed solution of monomer poly(ethylene glycol) methacrylate (PEGMA) and NapFFRK-acryloyl (peptidic hydrogelator), and the enzymes horseradish peroxidase (HRP), glucose oxidase (GOx), along with glucose, and acetyl acetone (AcAc), which commenced the two enzyme-mediated redox reaction. The hybrid hydrogel provided an enlarged time window for the in situ viscositycontrolled (of the pre-gel solution) printing into 3D constructs. NIH 3T3 cells-incorporated hybrid hydrogels exhibited a very high survival rate of $99.0 \pm 0.8 \%$, and a live/dead assay of the 3D bioprinted construct from such cell-laden bioink materials showed the cell viability of $98.0 \pm 2.3 \%$, suggesting the biocompatibilty of the hybrid hydrogels. The preparation of similar hybrid hydrogels by utilizing NapFFK-acryloyl hydrogelators (along with other components GOx, HRP, AcAc, and PEGMA) for their 3D cell printing was further illustrated by Wei et al. in another report. ${ }^{84}$ Such a supramolecular-polymeric hydrogel exhibited high mechanical strength with reusability and thermal stability along with biodegradability and cellular compatibility, and was successively employed for in situ 3D cellular printing to fabricate a 3D scaffold of NIH-3T3 cells.

A self-healable supramolecular polymer material for 3D printing under simplified thermomelting extrusion conditions was described by Wang et al. based on copolymer $\mathrm{P}(\mathrm{N}$-acryloyl glycinamide-co-1-vinyl-1,2,4-triazole) cross-linked through dynamic hydrogen bond interactions. ${ }^{85}$ The prepared hydrogels exhibited thermoplasticity, self-repairability, and reprocessability for various 3D printed constructs over a lower temperature range. By tuning the feed ratios and concentrations of monomer in the copolymer, the effective mechanical properties of the formed supramolecular hydrogels were obtained with high tensile strength up to $1.3 \mathrm{MPa}$, large stretchability up to more than $1300 \%$, and increased compressibility to $11 \mathrm{MPa}$ at swelling equilibrium state. Moreover, these supramolecular hydrogel biomaterials depicted medicinally desirable antibacterial and antiinflammatory activities, along with biocompatible properties.

Other notable systems include a 3D printed drug delivery implant developed by Roberts, Hayes, and coworkers based on thermo-responsive supramolecular polyurethane (SPU), able to form a self-assembled polymer network via hydrogen bonding and $\pi-\pi$ stacking (Fig. 6). ${ }^{86}$ The material displayed suitable mechanical properties (self-supporting and stiff, yet flexible) for the hot-melt extrusion-based approach at a relatively low processing temperature $\left(100{ }^{\circ} \mathrm{C}\right)$. To establish the potential application of SPU for the 3D printing of a biomedical device, the biocompatibility of the polymeric films of polyurethane was determined by MTT assay using mouse fibroblasts (L929), which illustrated their non-cytotoxicity with more than $94 \%$ of cell viability. A multicomponent synthetic material SPUPEG-paracetamol was prepared based on SPU co-formulated with PEG ( $4 \mathrm{wt} \%$ and $8 \mathrm{wt} \%$ ) and incorporating paracetamol drug $(16 \% \mathrm{w} / \mathrm{w})$ to afford 3D printed robust implant constructs that exhibited appropriate mechanical performance, and prolonged drug release over 5 to 8.5 months. Such release rates can be potentially altered by modulating the formulation with varying PEG percentage or molecular weight. In 2019, Binder et al. developed 3D printable inks based on supramolecular polymers as linear and three-arm star biocompatible poly(isobutylene)s exhibiting multiple hydrogen-bonds and their nanocomposites to enable the construction of stable and self-supported structures at room temperature with polymer filament diameters of $200-300 \mu \mathrm{m} .{ }^{87}$
(A)

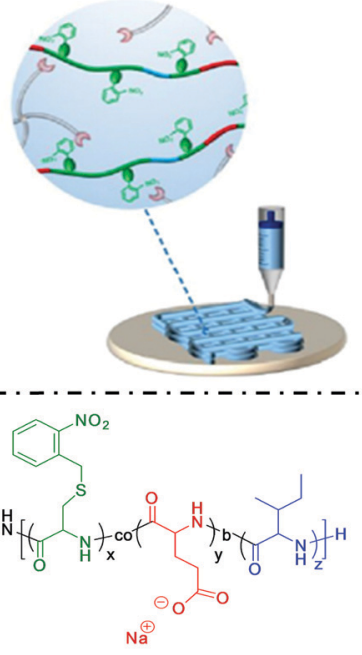

III

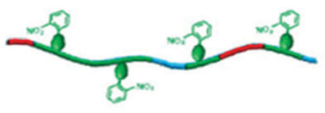

(B)

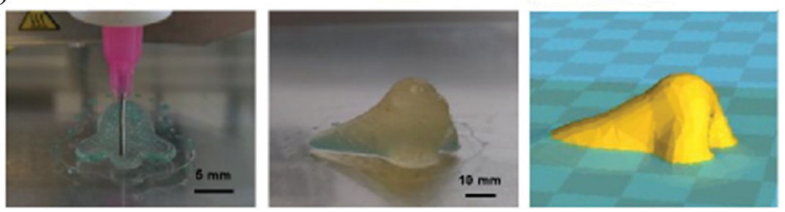

Fig. 8 (A) Schematic illustration of photoresponsive copolypeptide hydrogel-based 3D-extrusion printing. Photocleavage of nitrobenzyl protecting groups-generated free thiol residues, which further reacted with the alkyne functional groups of 4-arm PEG-propiolate. (B) The design and development of a human nose shape construct by 3D printing with hydrogel ink. Adapted from ref. 96 with permission from The Royal Society of Chemistry. 


\subsection{Stimuli-responsive polymers}

Stimuli-responsive polymers are smart or intelligent materials that can undergo alterations in their chemical, physical, morphological or mechanical properties upon interactions with their surrounding environment. ${ }^{88,89}$ In the past decade, these materials have been increasingly applied to a wide range of applications, including controlled and triggered drug delivery, (bio)sensing, actuators, coatings, diagnostics, tissue engineering, and biomedical devices. ${ }^{90,91}$ Various stimuli have been investigated for such polymers, including $\mathrm{pH}$, temperature, chemicals, light, redox, mechanical forces, biomolecules, electric fields, and magnetic fields. The synergistic integration of stimuli-responsive polymer materials with the 3D printing technique can potentially provide remarkable engineering and materials advancements in the field of additive manufacturing processes to create complex geometries and structures that are not possible via other manufacturing techniques. ${ }^{92,93}$ For instance, Pluronic F127 has been investigated as a stimuliresponsive ink material for $3 \mathrm{D}$ printing, which corresponds to the ABA-type triblock copolymer PEG-PPO-PEG and possesses inverse thermo-gelling properties, and can be readily printed and removed under mild conditions. ${ }^{8}$ Zhang et al. developed a similar type of hydrogel material with dual stimuliresponsiveness for 3D printing; however such ABA triblock copolymers were comprised of one hydrophilic PEG segment and two hydrophobic poly(isopropyl glycidyl ether) (PiPrGE) segments arranged in a PiPrGE-PEG-PiPrGE configuration, and the corresponding hydrogels were able to exhibit thermoreversibility, as well as a rapid and reversible response to shear forces. ${ }^{94}$ Hsieh et al. documented bioink materials based on the aqueous dispersions of thermoresponsive biodegradable polyurethane (PU) nanoparticles (comprised of diols of PCL and (L or D,L) PLA of $M_{\mathrm{n}} \sim 2000$, mixed in a 4:1 M ratio), which formed a hydrogel near $37{ }^{\circ} \mathrm{C}$ without using any crosslinker (Fig. 7). ${ }^{95}$ The hydrogel stiffness was optimized by varying the PU content ( 25 or $30 \%$ ) of the dispersion and their neural stem cell (NSC)-laden hydrogel constructs were printed by fused deposition manufacturing equipment. The filaments of the subsequently printed constructs displayed proper shape retention with reasonable swelling ( $<10 \%$ at $72 \mathrm{~h}$ ), e.g., from a diameter of $\sim 210 \mu \mathrm{m}$ at $0 \mathrm{~h}$ to $\sim 220 \mu \mathrm{m}$ after $72 \mathrm{~h}$ for PU2 (30\% or $25 \%$ ) hydrogels. The cell viability of the encapsulated NSCs in the above PU2 hydrogels $(\sim 680-2400 \mathrm{~Pa})$ was found to be greater than $100 \%$ at $72 \mathrm{~h}$, indicating excellent proliferation and differentiation. Moreover, the zebrafish embryo neural injury model having the injection with such NSCs (labeled with PKH26 (red fluorescence))-laden 25-30\% PU2 hydrogels exhibited their wide dispersion in all brain areas, in particular, for those with 25\% PU2 hydrogel, and further promoted the repair of the function of the impaired central nervous system. Murphy et al. illustrated another example of stimuli-responsive ink materials for 3D printing (Fig. 8) based on a photoresponsive polypeptide (with functional photoresponsive crosslinking). ${ }^{96}$ The hydrogel ink materials, comprised of UV-responsive copolypeptides incorporated with isolucine, glutamic acid, and cysteine (protected with photo-cleavable nitrobenzene groups),
(A)

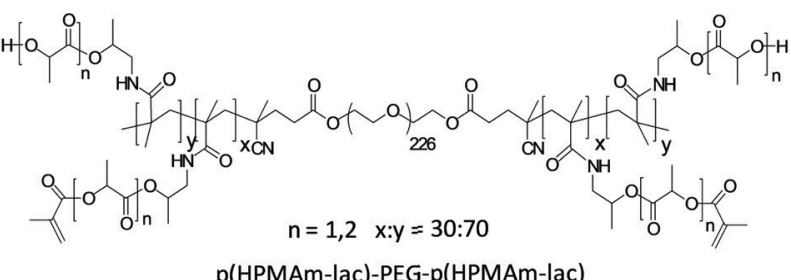

(B)

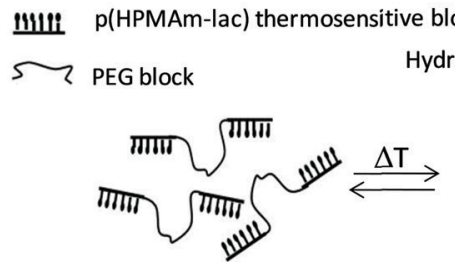

Sol

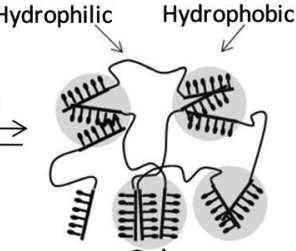

Gel
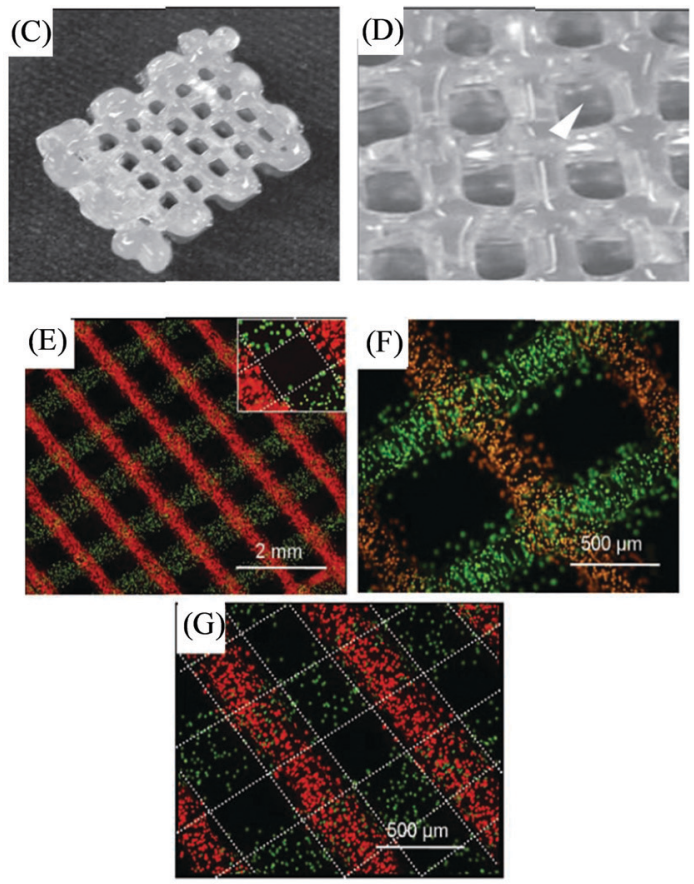

Fig. 9 (A) Chemical structure of the thermosensitive triblock copolymer. (B) A cartoon representation of the effect of temperature and photopolymerization on the polymer illustrating hydrogel formation above the cloud point followed by chemical cross-linking within the hydrophobic domain. (C and D) Photographs of the 3D printed construct from $25 \mathrm{wt} \%$ copolymer-based hydrogels with dimensions of $1 \times 1.5 \times 0.6 \mathrm{~cm}$, and strand spacing of $1.5 \mathrm{~mm}$. (E, F, and G) Microscopic images of the 3D printed layers of fluorescent microspheres-loaded hydrogels with $1.5 \mathrm{~mm}$ strand spacing of 2- and 3-layer angled constructs, and a $0.8 \mathrm{~mm}$ strand spacing of a 2-layer construct, respectively. Adapted with permission from ref. 97 . Copyright 2011 John Wiley and Sons. 
and alkyne functionalized 4-arm PEG-propiolate crosslinker, illustrated impressive shear-thinning properties to readily print a range of mechanically stable $3 \mathrm{D}$ structures. A catalyst-free nucleophilic thiol-yne "click" reaction was performed upon UV curing to enable crosslinking between the cysteine and propiolate residues, providing crosslinked hydrogel constructs with more than 10 layers exhibiting improved stiffness, high fidelity, and resolution. The biocompatibility of the copolypeptide hydrogel was further investigated with a human dermal fibroblasts cell assay by culturing on the top and within the hydrogel, illustrating almost no cytotoxicity and high viability of cells, respectively.

In 2011, Hennink and coworkers developed a biodegradable, photopolymerizable, and thermosensitive (as dual stimuliresponsive) ABA triblock copolymer hydrogel for 3D bioprinting-based cartilage tissue engineering applications (Fig. 9) ${ }^{97}$ A free radical copolymerization method was used to prepare this thermosensitive copolymer (with cloud point $\left.11{ }^{\circ} \mathrm{C}\right)$ comprised of A-blocks based on biodegradable $\operatorname{poly}(\mathrm{N}$ (2-hydroxypropyl)methacrylamide lactate) (p(HPMAm-lac)) $\left(M_{\mathrm{W}} \sim 23.5 \mathrm{kDa}\right)$ partially modified by methacrylate groups to access photo cross-linking, and B-block derived from the hydrophilic PEG $\left(M_{\mathrm{w}} \sim 10 \mathrm{kDa}\right)$, yielding p(HPMA-lac)-PEG$\mathrm{p}$ (HPMA-lac). The as-developed p(HPMA-lac)-PEG-p(HPMAlac)-based hydrogels, with thermally and chemically crosslinked networks, displayed strain-softening behaviour and illustrated semi-flexible properties similar to various natural polymers including collagen. Moreover, the copolymeric hydrogels (25 wt\%) were found to be suitable for the printing of porous $3 \mathrm{D}$ constructs with subsequent photopolymerization to exhibit an elastic modulus of $119 \mathrm{kPa}$ (for the $0.6 \mathrm{~cm}$ construct) and a degradation time of around 190 days (for the 12-layered construct), and offer mechanical support to the encapsulated cells for the long term until new tissue is formed. Different fluorescent microspheres (fluorescent orange and lemon)loaded hydrogels displayed the accurate and precise localization of such cell mimicking microspheres encapsulated within the copolymer to their 3D fiber deposition. The cell-laden photopolymerized hydrogel (25 wt\%) revealed the homogeneous distribution of chondrocytes over the entire hydrogel with excellent viability of $94 \%$ after 1 day, with no adverse effects found due to the exposure of UV.

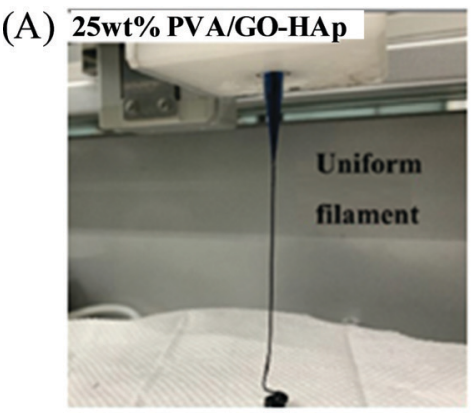

(B)

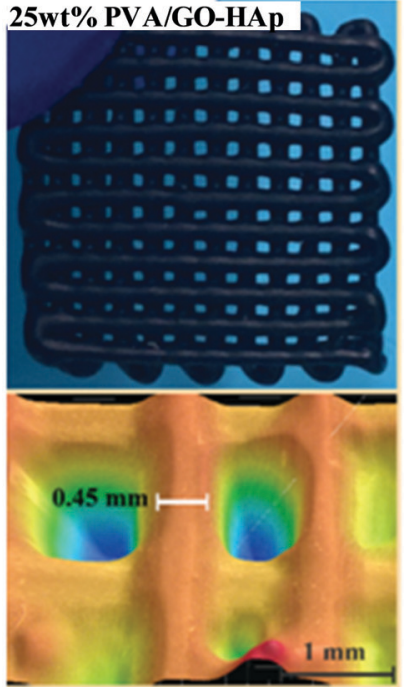

(C)

(D)
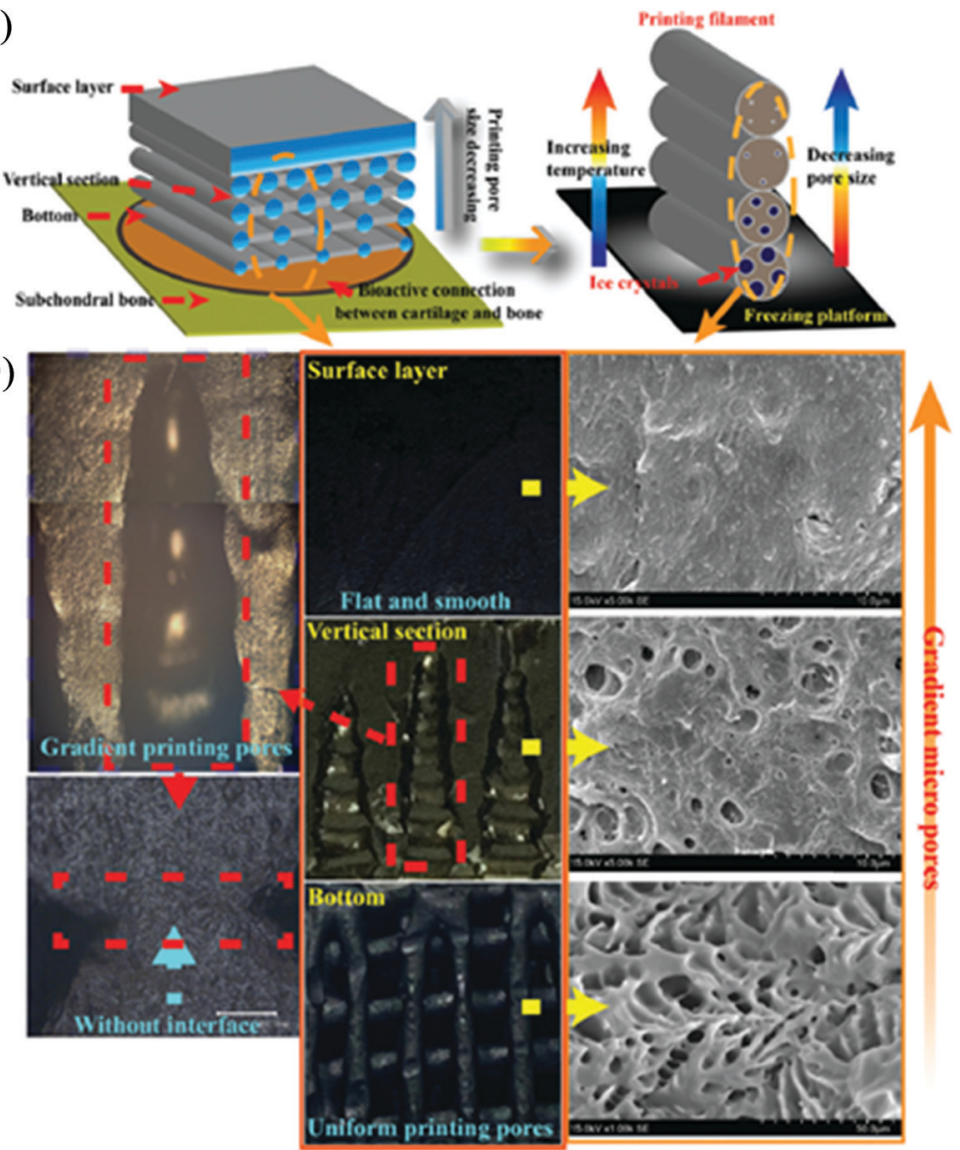

Fig. 10 (A) Digital photograph of a 3D printing filament of the PVA/GO-HAp nanocomposite solution with 25 wt\% PVA concentration. (B) Digital photograph and structural morphology of the 3D printed sample for the above nanocomposite-based hydrogel having a reticular porous structure. (C) Schematic design of the PVA/GO-HAp nanocomposite-based biomimetic gradient porous structure as artificial cartilage. (D) Digital photograph and SEM images of the 3D printed constructs of PVA/GO-HAp (25 wt\%) nanocomposite-based artificial cartilage. Adapted from ref. 104 with permission from The Royal Society of Chemistry. 
(A)

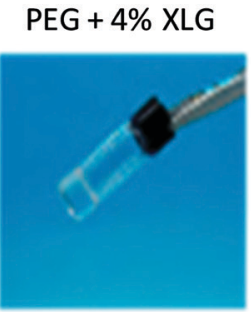

(B)

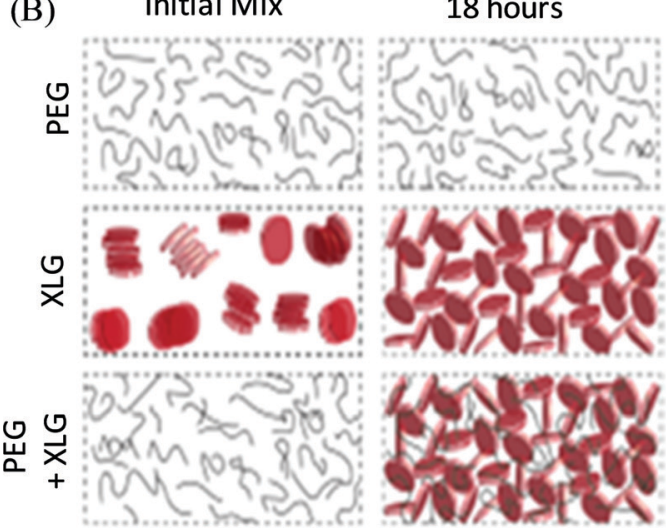

(C)

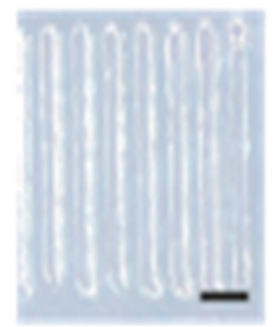

18 hours
(D)
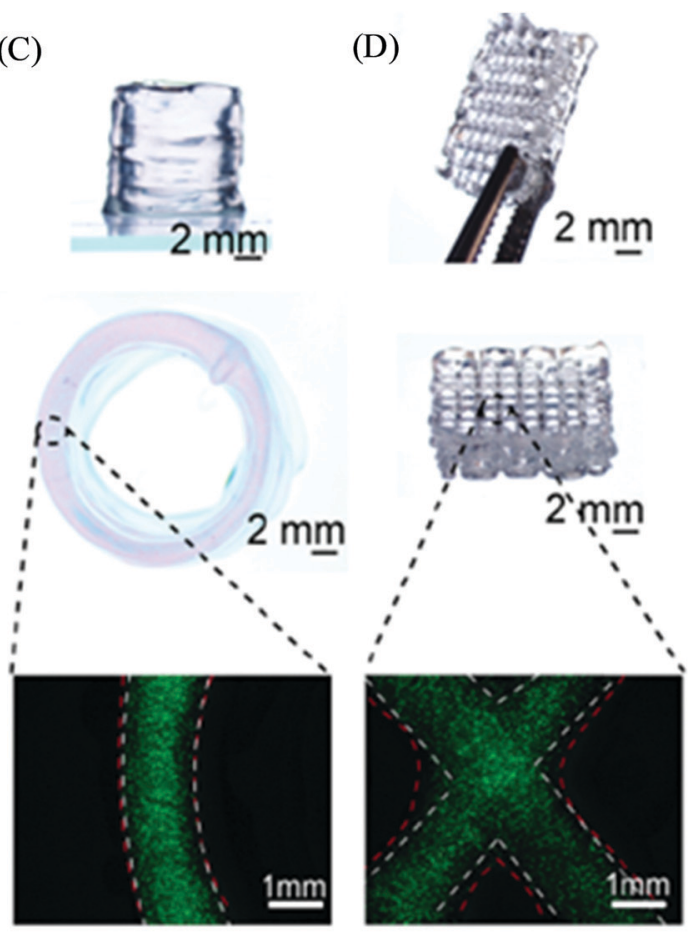

Fig. 11 (A) Digital photograph of the PEG-LAPONITE ${ }^{\mathbb{R}}$ colloidal solution-based hydrogel and their printed structure. (B) Schematic of the formation of the internal structure for PEG, LAPONITE ${ }^{\circledR}$, and PEG-LAPONITE ${ }^{\circledR}$ nanocomposite solutions at the initial time and after 18 hours. (C and D) 3D printed circular and crosshatch structure, respectively, of PEG-(4\%) LAPONITE ${ }^{\mathbb{R}}$ with preosteoblasts and cell tracker images. Adapted with permission from ref. 106. Copyright 2018 American Chemical Society.

\subsection{Nanoengineered polymers}

Nanoengineered polymers are hybrid materials in which a polymeric matrix is dispersed with one or more types of inorganic or organic nanofillers such as silica or metallic nanoparticles, nanoclay, nanorods, carbon nanotubes and nanofibers, graphene, metallic nanowires, quantum dots, etc. ${ }^{98,99}$ Due to the several advantages imparted by the incorporated nanomaterials (via choosing proper mixing strategies) within the polymer matrix, such fabricated polymer nanocomposite materials exhibit significantly improved rheological performances, functionalities and properties (e.g., mechanical, electrical, optical, thermal, biological and magnetic properties) with respect to the native polymers, ${ }^{100,101}$ and therefore, make the polymer nanocomposite a suitable candidate to enable additive manufacturing based highlycustomized complex and functional 3D constructs. ${ }^{102,103}$ Meng et al. documented a poly(vinyl alcohol) (PVA)-based nanocomposite hydrogel as artificial cartilage constructed by extrusion-based 3D printing (Fig. 10). ${ }^{104}$ Incorporation of graphene oxide (GO)hydroxyapatite (HAp) in the nanocomposite PVA/GO-HAp (25 wt\%) highly improved the dynamic viscosity of the materials and exhibited good shear-thinning properties in the extrusion shear rate range with a reduced Barus effect in order to obtain the stable printed constructs with high printing accuracy. The 3D printed PVA/GO-HAp hydrogels displayed biomimetic gradient porous structures with excellent bio-mechanical and bio-frictional behaviors attributed to their promising potential for the exact repair of articular cartilage. Shah and coworkers demonstrated the extrusion-based 3D printing of polymer nanocomposite inks comprised of a biocompatible copolymer polylactide-co-glycolide (PLG) (85:15) with high content of graphene (60 vol\% of solid) to construct a mechanically robust and flexible scaffold (with strands ranging from 100 to $1000 \mu \mathrm{m}$ in diameter) with shape-fidelity and enhanced electrical conductivities $>800 \mathrm{~S} \mathrm{~m}^{-1}$ for electronic applications. $^{105}$ The printed 3D scaffold further revealed their potential biomedical application towards nerve tissue engineering and regeneration by exhibiting their significant cellular response (attachment, proliferation, and neurogenic differentiation) to human mesenchymal stem cells (hMSCs) and viability to multiple, distinct cell types, including hMSCs.

Polymer/clay nanocomposite ink materials based on PEG precursor colloidal solutions incorporated with biocompatible and bioactive disk-shaped 2D LAPONITE ${ }^{\circledR}$ (XLG) nanoclay were investigated by the Gaharwar group for 3D bioprinting (Fig. 11). ${ }^{106}$ Such hydrogels based on diacrylated PEG-LAPONITE ${ }^{\circledR}$ demonstrated shear-thinning and self-healing properties due to rapid internal phase rearrangement, thus enabling the printing of a range of complex 3D structures upon UV-based photocrosslinking. The rheological characteristic of the nanocomposite hydrogels appeared to be independent of the addition of PEG and dominated by the behaviour of the LAPONITE ${ }^{\circledR}$ network, whereas their self-recovery time was found to be controlled by the ratio of PEG:LAPONITE ${ }^{\circledR}$. Further, the PEG-(4\%)LAPONITE ${ }^{\circledR}$-based bioinks exhibited high cell viability of the encapsulated murine preosteoblasts immediately post-injection, and the viability was further maintained across different volumetric flow rates of 500, 1000 , and $200 \mu \mathrm{L} \mathrm{min}{ }^{-1}$ as indicated by the live/dead assay. 
Another example of nanoclay-based polymeric nanocomposite bioink for 3D printing was demonstrated by Zhai et al., derived from supramolecular polymer poly( $N$-acryloyl glycinamide $)$ (PNAGA). ${ }^{107}$ The polymer/clay PNAGA-LAPONITE ${ }^{\mathbb{R}}$ XLG-based hybrid hydrogel ink provided the fabrication of a 3D high strength composite scaffold with swelling stability (via post-extrusion UV light irradiation) imparted by the dual amide hydrogen bonding interactions of polymer combined with physical cross-linking of nanoclay-polymer chain interactions. Such PNAGA(20\%)LAPONITE $^{\mathbb{R}}$ bioscaffolds were able to promote the osteogenic differentiation of primary rat osteoblast (ROB) cells via the release of encapsulated $\mathrm{Mg}^{2+}$ and $\mathrm{Si}^{4+}$ ions from the hydrogel as suggested by the in vitro studies. Importantly, improved cellular adhesion, proliferation, and differentiation bioactivities were observed for ROB seeded within the PNAGA-LAPONITE ${ }^{\circledR}$ composite scaffolds due to the incorporation of clay into such hydrogel systems. The in vivo experiments demonstrated the efficient formation of new bone upon the implantation of the PNAGA(20\%)-clay scaffold into tibia defects of rats for 8 weeks.

\section{Conclusions and future perspectives}

The field of $3 \mathrm{D}$ printing-based additive manufacturing has been burgeoning over the past few years and a range of affordable 3D printers are accessible for laboratories to date. A lot of efforts have been devoted to this field, essentially towards (i) the engineering and optimization of the printing techniques for enabling the creation of complex and highly organised structures with controlled processing and higher resolution, and (ii) developing the ink materials with suitable rheological properties and functionality. The particular challenge is the development of (bio)ink materials for the $3 \mathrm{D}$ printing of biologically relevant scaffolds/tissue replacement, which allows the cells to adhere, differentiate and proliferate, and preserves the bioactivity of their other embedded compounds such as growth factors, signalling peptides, drugs, etc. Currently, researchers are focusing on the design of (bio)ink materials mainly based on natural polymers or a combination of natural and synthetic polymers; however, this approach is associated with several challenges, and therefore, the field of $3 \mathrm{D}$ (bio)printing is still in its infancy. The introduction of synthetic polymers as a single (or major) network precursor of ink, and the involvement of the functionalization and cross-linking chemistry in the ink formulation can lead to the development of a diverse range of new-generation (bio)ink materials with tuneable mechanical, chemical and biological properties, and can therefore emerge as a key player for advancing the field of $3 \mathrm{D}$ printing to the next frontier to create synthetic and inhuman $3 \mathrm{D}$ printed tissues. It is critically important to have a fundamental understanding of engineering the 3D printing technique with controlled processing parameters, evaluating new and advanced polymers-based ink/bioink materials, and designing the biomimetic hard and soft tissue scaffolds with controlled structural integrity and functioning to fabricate the targeted tissue/organ; this will drive further innovation in the field of tissue engineering and regenerative medicines.

\section{Conflicts of interest}

The authors declare no conflict of interest.

\section{Acknowledgements}

The author acknowledge the financial support by Science and Engineering Research Board (SERB), New Delhi, India (Project no.: SRG/2020/000610).

\section{References}

1 R. L. Truby and J. A. Lewis, Nature, 2016, 540, 371-378.

2 (a) S. C. Ligon, R. Liska, J. Stampfl, M. Gurr and R. Mulhaupt, Chem. Rev., 2017, 117, 10212-10290; (b) D. Gräfe, S. L. Walden, J. Blinco, M. Wegener, E. Blasco and C. Barner-Kowollik, Angew. Chem., Int. Ed., 2020, 59, 6330-6340.

3 (a) S. H. Park, C. S. Jung and B. H. Min, Tissue Eng. Regener. Med., 2016, 13, 622-635; (b) A. Shapira and T. Dvir, Adv. Sci., 2021, 8, 2003751.

4 (a) A. Lee, A. R. Hudson, D. J. Shiwarski, J. W. Tashman, T. J. Hinton, S. Yerneni, J. M. Bliley, P. G. Campbell and A. W. Feinberg, Science, 2019, 365, 482-487; (b) M. S. Mannoor, Z. Jiang, T. James, Y. L. Kong, K. A. Malatesta, W. O. Soboyejo, N. Verma, D. H. Gracias and M. C. McAlpine, Nano Lett., 2013, 13, 2634-2639.

5 M. Nadgorny and A. Ameli, ACS Appl. Mater. Interfaces, 2018, 10, 17489-17507.

6 S. Naficy, R. Gately, R. Gorkin, H. Xin and G. M. Spinks, Macromol. Mater. Eng., 2017, 302, 1600212.

7 H. Gudapati, M. Dey and I. Ozbolat, Biomaterials, 2016, 102, 20-42.

8 D. B. Kolesky, R. L. Truby, A. S. Gladman, T. A. Busbee, K. A. Homan and J. A. Lewis, Adv. Mater., 2014, 26, 3124-3130.

9 B. Holmes, K. Bulusu, M. Plesniak and L. G. Zhang, Nanotechnology, 2016, 27, 064001.

10 R. J. Mondschein, A. Kanitkar, C. B. Williams, S. S. Verbridge and T. E. Long, Biomaterials, 2017, 140, 170-188.

11 C. M. B. Ho, A. Mishra, K. Hu, J. An, Y.-J. Kim and Y.-J. Yoon, ACS Biomater. Sci. Eng., 2017, 3, 2198-2214.

12 T. Jungst, W. Smolan, K. Schacht, T. Scheibel and J. Groll, Chem. Rev., 2016, 116, 1496-1539.

13 J. M. Lee and W. Y. Yeong, Adv. Healthcare Mater., 2016, 5, 2856-2865.

$14 \mathrm{H}$. Abe, K. Hayashi and M. Sato, Data book on mechanical properties of living cells tissues, and organs, Springer, 1996.

15 S. S. Athukorala, T. S. Tran, R. Balu, V. K. Truong, J. Chapman, N. K. Dutta and N. R. Choudhury, Polymers, 2021, 13, 474 . 
16 L. Li, Q. Lin, M. Tang, A. J. E. Duncan and C. Ke, Chem. Eur. J., 2019, 25, 1-15.

17 L. Valot, J. Martinez, A. Mehdi and G. Subra, Chem. Soc. Rev., 2019, 48, 4049-4086.

18 F. You, X. Wu, N. Zhu, M. Lei, B. F. Eames and X. Chen, ACS Biomater. Sci. Eng., 2016, 2, 1200-1210.

19 J. Y. Park, G. Gao, J. Jang and D.-W. Cho, J. Mater. Chem. B, 2016, 4, 7521-7539.

20 V. Mourino and A. R. Boccaccini, J. R. Soc., Interface, 2010, 7, 209-227.

21 I. T. Ozbolat, W. Peng and V. Ozbolat, Drug Discovery Today, 2016, 21, 1257-1271.

22 Y. He, F. F. Yang, H. M. Zhao, Q. Gao, B. Xia and J. Z. Fu, Sci. Rep., 2016, 6, 29977.

23 A. Schwab, R. Levato, M. D’Este, S. Piluso, D. Eglin and J. Malda, Chem. Rev., 2020, 120, 11028-11055.

24 M. Bongio, J. J. van den Beucken, M. R. Nejadnik, S. C. Leeuwenburgh, L. A. Kinard, F. K. Kasper, A. G. Mikos and J. A. Jansen, Eur. Cell. Mater., 2011, 22, 359.

25 (a) K. Y. Lee, M. C. Peters, K. W. Anderson and D. J. Mooney, Nature, 2000, 408, 998; (b) R. Censi, P. Di Martino, T. Vermonden and W. E. Hennink, J. Controlled Release, 2012, 161, 680.

26 K. L. Spiller, S. A. Maher and A. M. Lowman, Tissue Eng., Part B, 2011, 17, 281.

27 H. Gudapati, M. Dey and I. Ozbolat, Biomaterials, 2016, 102, 20-42.

28 I. Freshney, Culture of Animal Cells: A Manual of Basic Technique and Specialized Applications, John Wiley \& Sons, 2015.

29 K. Nair, M. Gandhi, S. Khalil, K. C. Yan, M. Marcolongo, K. Barbee and W. Sun, Biotechnol. J., 2009, 4, 1168-1177.

30 J. Liu, L. Sun, W. Xu, Q. Wang, S. Yu and J. Sun, Carbohydr. Polym., 2019, 207, 297-316.

31 C. Mota, S. Camarero-Espinosa, M. B. Baker, P. Wieringa and L. Moroni, Chem. Rev., 2020, 120, 10547-10607.

32 G. Ratheesh, J. R. Venugopal, A. Chinappan, H. Ezhilarasu, A. Sadiq and S. Ramakrishna, ACS Biomater. Sci. Eng., 2017, 3, 1175-1194.

33 M. Guvendiren, J. Molde, R. M. Soares and J. Kohn, ACS Biomater. Sci. Eng., 2016, 2, 1679-1693.

34 S. R. Govindarajan, Y. Xu, J. P. Swanson, T. Jain, Y. Lu, J.-W. Choi and A. Joy, Macromolecules, 2016, 49, 2429-2437.

35 D. A. Gopakumar, D. Pasquini, M. A. Henrique, L. C. de Morais, Y. Grohens and S. Thomas, ACS Sustainable Chem. Eng., 2017, 5, 2026-2033.

36 L.-Y. Zhou, J. Fu and Y. He, Adv. Funct. Mater., 2020, 30, 2000187.

37 B. Narupai and A. Nelson, ACS Macro Lett., 2020, 9, 627-638.

38 R. R. Jose, M. J. Rodriguez, T. A. Dixon, F. Omenetto and D. L. Kaplan, ACS Biomater. Sci. Eng., 2016, 2, 1662-1678.

39 C.-C. Lin and K. S. Anseth, Pharm. Res., 2009, 26, 631-643. 40 C. Englert, J. C. Brendel, T. C. Majdanski, T. Yildirim, S. Schubert, M. Gottschaldt, N. Windhab and U. S. Schubert, Prog. Polym. Sci., 2018, 87, 107-164.
41 L. Tan, X. Yu, P. Wan and K. Yang, J. Mater. Sci. Technol., 2013, 29, 503-513.

42 M. K. Gupta, F. Meng, B. N. Johnson, Y. L. Kong, L. Tian, Y.-W. Yeh, N. Masters, S. Singamaneni and M. C. McAlpine, Nano Lett., 2015, 15, 5321-5329.

43 P. Camacho, H. Busari, K. Seims, P. Schwarzenberg, H. L. Dailey and L. W. Chow, Biomater. Sci., 2019, 7, 4237-4247.

44 A. Rashad, S. Mohamed-Ahmed, M. Ojansivu, K. Berstad, M. A. Yassin, T. Kivijarvi, E. B. Heggset, K. Syverud and K. Mustafa, Biomacromolecules, 2018, 19, 4307-4319.

45 W. J. Peng, D. Unutmaz and I. T. Ozbolat, Trends Biotechnol., 2016, 34, 722-732.

46 M. Askari, M. A. Naniz, M. Kouhi, A. Saberi, A. Zolfagharian and M. Bodaghi, Biomater. Sci., 2021, 9, 535-573.

47 J. Malda, J. Visser, F. P. Melchels, T. Jungst, W. E. Hennink, W. J. Dhert, J. Groll and D. W. Hutmacher, Adv. Mater., 2013, 25, 5011-5028.

48 Z. L. Yue, X. Liu, P. T. Coates and G. G. Wallace, Curr. Opin. Organ Transplant., 2016, 21, 467-475.

49 A. C. Fonseca, F. P. W. Melchels, M. J. S. Ferreira, S. R. Moxon, G. Potjewyd, T. R. Dargaville, S. J. Kimber and M. Domingos, Chem. Rev., 2020, 120, 11093-11139.

50 X. Cui, J. Li, Y. Hartanto, M. Durham, J. Tang, H. Zhang, G. Hooper, K. Lim and T. Woodfield, Adv. Healthcare Mater., 2020, 1901648.

51 Z. Jiang, B. Diggle, M. L. Tan, J. Viktorova, C. W. Bennett and L. A. Connal, Adv. Sci., 2020, 7, 2001379.

52 L. Ouyang, C. B. Highley, W. Sun and J. A. Burdick, Adv. Mater., 2017, 1604983.

53 A. McCormack, C. B. Highley, N. R. Leslie and F. P. W. Melchels, Trends Biotechnol., 2020, 38, 584-593.

54 J. J. Senior, M. E. Cooke, L. M. Grover and A. M. Smith, Adv. Funct. Mater., 2019, 29, 1904845.

55 E. Mirdamadi, J. W. Tashman, D. J. Shiwarski, R. N. Palchesko and A. W. Feinberg, ACS Biomater. Sci. Eng., 2020, 6, 6453-6459.

56 E. Davoodi, E. Sarikhani, H. Montazerian, S. Ahadian, M. Costantini, W. Swieszkowski, S. M. Willerth, K. Walus, M. Mofidfar, E. Toyserkani, A. Khademhosseini and N. Ashammakhi, Adv. Mater. Technol., 2020, 5, 1901044.

57 C. Colosi, S. R. Shin, V. Manoharan, S. Massa, M. Costantini, A. Barbetta, M. R. Dokmeci, M. Dentini and A. Khademhosseini, Adv. Mater., 2016, 28, 677-684.

58 (a) S. J. Buwalda, T. Vermonden and W. E. Hennink, Biomacromolecules, 2017, 18, 316; (b) R. Dong, Y. Pang, Y. Su and X. Zhu, Biomater. Sci., 2015, 3, 937.

59 (a) A. M. Jonker, D. W. P. M. Lowik and J. C. M. van Hest, Chem. Mater., 2012, 24, 759; (b) S. Kumar and W. H. Binder, Soft Matter, 2020, 16, 6964-6968.

60 J. E. Kim, S. H. Kim and Y. Jung, Tissue Eng. Regener. Med., 2016, 13, 636-646.

61 C. D. Spicer, Polym. Chem., 2020, 11, 184-219.

62 J. M. Unagolla and A. C. Jayasuriya, Appl. Mater. Today, 2020, 18, 100479. 
63 R. Censi, W. Schuurman, J. Malda, G. di Dato, P. E. Burgisser, W. J. A. Dhert, C. F. van Nostrum, P. di Martino, T. Vermonden and W. E. Hennink, Adv. Funct. Mater., 2011, 21, 1833.

64 (a) N. E. Fedorovich, J. R. De Wijn, A. J. Verbout, J. Alblas and W. J. Dhert, Tissue Eng., Part A, 2008, 14, 127; (b) S. Hong, D. Sycks, H. F. Chan, S. Lin, G. P. Lopez, F. Guilak, K. W. Leong and X. Zhao, Adv. Mater., 2015, 15, 4035-4040.

65 N. E. Fedorovich, I. Swennen, J. Girones, L. Moroni, C. A. van Blitterswijk, E. Schacht, J. Alblas and W. J. Dhert, Biomacromolecules, 2009, 10, 1689.

66 (a) C. M. Smith, A. L. Stone, R. L. Parkhill, R. L. Stewart, M. W. Simpkins, A. M. Kachurin, W. L. Warren and S. K. Williams, Tissue Eng., 2004, 10, 1566; (b) A. C. Daly, G. M. Cunniffe, B. N. Sathy, O. Jeon, E. Alsberg and D. J. Kelly, Adv. Healthcare Mater., 2016, 5, 2353-2362.

67 H. W. Ooi, C. Mota, A. T. ten Cate, A. Calore, L. Moroni and M. B. Baker, Biomacromolecules, 2018, 19, 3390-3400.

68 A. Skardal, J. Zhang and G. D. Prestwich, Biomaterials, 2010, 31, 6173-6181.

69 S. Joas, G. E. M. Tovar, O. Celik, C. Bonten and A. Southan, Gels, 2018, 4, 69.

70 C. Xu, W. Lee, G. Dai and Y. Hong, ACS Appl. Mater. Interfaces, 2018, 10, 9969-9979.

71 L. Brunsveld, B. Folmer, E. Meijer and R. Sijbesma, Chem. Rev., 2001, 101, 4071-4098.

72 (a) E. Kolomiets and J.-M. Lehn, Chem. Commun., 2005, 1519; (b) S. Kumar, V. Bheemireddy and P. De, Macromol. Biosci., 2015, 15, 1447-1456.

73 T. Aida, E. W. Meijer and S. I. Stupp, Science, 2012, 335, 813.

74 (a) J. L. Mann, A. C. Yu, G. Agmon and E. A. Appel, Biomater. Sci., 2018, 6, 10-37; (b) S. Kumar, R. Acharya, U. Chatterji and P. De, Polym. Chem., 2014, 5, 6039-6050.

75 C. F. Faul and M. Antonietti, Adv. Mater., 2003, 15, 673-683.

76 E. R. Johnson, S. Keinan, P. Mori-Sanchez, J. ContrerasGarcia, A. J. Cohen and W. Yang, J. Am. Chem. Soc., 2010, 132, 6498-6506.

77 X. Yan, F. Wang, B. Zheng and F. Huang, Chem. Soc. Rev., 2012, 41, 6042-6065.

78 S.-L. Li, T. Xiao, C. Lin and L. Wang, Chem. Soc. Rev., 2012, 41, 5950-5968.

79 A. M. Pekkanen, R. J. Mondschein, C. B. Williams and T. E. Long, Biomacromolecules, 2017, 18, 2669-2687.

80 C. Echalier, R. Levato, M. A. Mateos-Timoneda, O. Castaño, S. Déjean, X. Garric, C. Pinese, D. Noël, E. Engel, J. Martinez, A. Mehdi and G. Subra, RSC Adv., 2017, 7, 12231-12235.

81 T. Lorson, S. Jaksch, M. M. Lubtow, T. Jungst, J. Groll, T. Luhmann and R. Luxenhofer, Biomacromolecules, 2017, 18, 2161-2171.

82 L. Trachsel, M. Zenobi-Wong and E. M. Benetti, Biomater. Sci., 2021, 9, 2874-2886.

83 Q. Wei, W. Xu, M. Liu, Q. Wu, L. Cheng and Q. Wang, J. Mater. Chem. B, 2016, 4, 6302-6306.
84 Q. Wei, M. Xu, C. Liao, Q. Wu, M. Liu, Y. Zhang, C. Wu, L. Cheng and Q. Wang, Chem. Sci., 2016, 7, 2748-2752.

85 H. Wang, H. Zhu, W. Fu, Y. Zhang, B. Xu, F. Gao, Z. Cao and W. Liu, Macromol. Rapid Commun., 2017, 38, 1600695.

86 S. Salimi, Y. Wu, M. I. E. Barreiros, A. A. Natfji, S. Khaled, R. Wildman, L. R. Hart, F. Greco, E. A. Clark, C. J. Roberts and W. Hayes, Polym. Chem., 2020, 11, 3453-3464.

87 H. Rupp, D. Döhler, P. Hilgeroth, N. Mahmood, M. Beiner and W. H. Binder, Macromol. Rapid Commun., 2019, 40, 1900467.

88 M. A. C. Stuart, W. T. S. Huck, J. Genzer, M. Müller, C. Ober, M. Stamm, G. B. Sukhorukov, I. Szleifer, V. V. Tsukruk, M. Urban, F. Winnik, S. Zauscher, I. Luzinov and S. Minko, Nat. Mater., 2010, 9, 101-113.

89 (a) Y. Lu, A. A. Aimetti, R. Langer and Z. Gu, Nat. Rev. Mater., 2017, 2, 16075; (b) S. Kumar, S. Deike and W. H. Binder, Macromol. Rapid Commun., 2018, 39, 1700507.

90 M. Wei, Y. Gao, X. Li and M. J. Serpe, Polym. Chem., 2017, 8, 127-143.

91 T. M. Reineke, ACS Macro Lett., 2016, 5, 14-18.

92 (a) A. J. Boydston, B. Cao, A. Nelson, R. J. Ono, A. Saha, J. J. Schwartz and C. J. Thrasher, J. Mater. Chem. A, 2018, 6, 20621-20645; (b) H. Rupp and W. H. Binder, Macromol. Rapid Commun., 2020, 42, 2000450.

93 G. I. Peterson, M. B. Larsen, M. A. Ganter, D. W. Storti and A. J. Boydston, ACS Appl. Mater. Interfaces, 2015, 7, 577-583.

94 M. Zhang, A. Vora, W. Han, R. J. Wojtecki, H. Maune, A. B. A. Le, L. E. Thompson, G. M. McClelland, F. Ribet, A. C. Engler and A. Nelson, Macromolecules, 2015, 48, 6482-6488.

95 F. Y. Hsieh, H. H. Lin and S. H. Hsu, Biomaterials, 2015, 71, 48-57.

96 R. D. Murphy, S. Kimmins, A. J. Hibbitts and A. Heise, Polym. Chem., 2019, 10, 4675-4682.

97 R. Censi, W. Schuurman, J. Malda, G. di Dato, P. E. Burgisser, W. J. A. Dhert, C. F. van Nostrum, P. di Martino, T. Vermonden and W. E. Hennink, Adv. Funct. Mater., 2011, 21, 1833-1842.

98 S. K. Kumar, B. C. Benicewicz, R. A. Vaia and K. I. Winey, Macromolecules, 2017, 50, 714-731.

99 D. A. Gopakumar, A. R. Pai, Y. B. Pottathara, D. Pasquini, L. C. de Morais, M. Luke, N. Kalarikkal, Y. Grohens and S. Thomas, ACS Appl. Mater. Interfaces, 2018, 10, 20032-20043.

100 B. Elder, R. Neupane, E. Tokita, U. Ghosh, S. Hales and Y. L. Kong, Adv. Mater., 2020, 32, 1907142.

101 H. Rupp and W. H. Binder, Adv. Mater. Technol., 2020, 2000509. 102 R. D. Farahani and M. Dubé, Adv. Eng. Mater., 2018, 20, 1700539.

103 H. Rastin, B. Zhang, A. Mazinani, K. Hassan, J. Bi, T. Tung and D. Losic, Nanoscale, 2020, 12, 16069-16080.

104 Y. Meng, J. Cao, Y. Chen, Y. Yu and L. Ye, J. Mater. Chem. B, 2020, 8, 677-690.

105 A. E. Jakus, E. B. Secor, A. L. Rutz, S. W. Jordan, M. C. Hersam and R. N. Shah, ACS Nano, 2015, 9, 4636-4648.

106 C. W. Peak, J. Stein, K. A. Gold and A. K. Gaharwar, Langmuir, 2018, 34, 917-925.

107 X. Zhai, Y. Ma, C. Hou, F. Gao, Y. Zhang, C. Ruan, H. Pan, W. W. Lu and W. Liu, ACS Biomater. Sci. Eng., 2017, 3, 1109-1118. 